\title{
Garantía de Calidad de Cualificaciones Intermedias de la Industria Farmacéutica
}

\author{
Quality Assurance of Intermediate Qualifications in the Pharmaceutical Industry
}

Garantia de Qualidade de Qualificações Intermediárias da Indústria Farmacéutica

Francisca Arbizu Echavarri* https://orcid.org/0000-0003-4872-7551

Catedrática de Educación Secundaria

$\checkmark$

Recibido: 06-05-18 Revisado: 15-06-188 Aceptado: 15-10-18 Publicado: 20-12-18

- Resumen. La investigación recogida en la Tesis "Cualificaciones profesionales de la industria farmacéutica en el marco de la LOGSE" responde a la necesidad de comprobar la pertinencia, calidad y efectividad de los títulos de Técnico en Operaciones de Fabricación de Productos Farmacéuticos, (TOFPF), en el nivel secundario y de Técnico Superior en Fabricación de Productos Farmacéuticos y Afines (TSFPA), en la educación superior. Ambos se crean en 1993, para responder a directivas europeas y legislación española, desde 1991, sobre Normas de Correcta Fabricación de medicamentos, NCF, de uso humano, veterinario y de cosméticos, los cuales exigen que los fabricantes dispongan de suficiente personal competente, con las cualificaciones adecuadas en todas sus instalaciones de fabricación para alcanzar el objetivo de garantía de la calidad farmacéutica. El artículo recoge la evaluación de la calidad del título TSFPA desde sus mecanismos de identificación y diseño (1991-1994), así como de los resultados y efectos de su implantación y desarrollo (1995-2015), e incluso su revisión (20042017) y su impacto aportando evidencias empíricas cualitativas y cuantitativas, de acuerdo con los indicadores de referencia propuestos por la Recomendación sobre el establecimiento de un Marco de Referencia Europeo de Garantía de la Calidad en la FP.
Palabras clave:

Cualificación,

formación

profesional,

calidad,

evaluación,

industria

farmacéutica 
Abstract. This research-included in the "Professional Qualifications of the Pharmaceutical Industry Within the Framework of the LOGSE" thesis-responds to the need to verify the relevance, quality, and effectiveness of the degrees of Technician in Manufacturing Operations of Pharmaceutical Products, (TOFPF), at the secondary level, and Higher Technician in Manufacturing of Pharmaceutical and Related Products (TSFPA), in higher education. Both degrees were created in 1993, in response to European Directives and Spanish legislation, dated as of 1991, on Good Manufacturing Practices of Medications, for human, veterinary, and cosmetics use, which requires manufacturers to have sufficient competent staff, with appropriate qualifications in all their manufacturing facilities to achieve the objective of pharmaceutical quality assurance. The article includes the evaluation of the quality of the TSFPA degree from its identification and design mechanisms (1991-1994), as well as the results and effects of its implementation and development (1995-2015), and even its revision (2004-2017) and impact providing qualitative and quantitative empirical evidence, according to the reference indicators proposed by the Recommendation on the establishment of a European Framework of Reference for Quality Assurance in Professional Training.

- Resumo. A pesquisa coletada na Tese “Qualificações profissionais da indústria farmacêutica no âmbito da LOGSE" responde à necessidade de comprovar a pertinência, qualidade e efetividade dos títulos de Técnico em Operações de Fabricação de Produtos Farmacêuticos, (TOFPF), no nível secundário e de Técnico Superior em Fabricação de Produtos Farmacêuticos e Afins (TSFPA), no ensino superior. Ambos foram criados no ano 1993 para responder às Diretivas europeias e a legislação espanhola, desde 1991, sobre Normas de Correta Fabricação de medicamentos, NCF, de uso humano, veterinário e de cosméticos, que exige que os fabricantes disponham de suficiente pessoal competente, com qualificações adequadas em todas as instalações de fabricação para atingir o objetivo de garantia da qualidade farmacêutica. O artigo reúne a avaliação da qualidade do título TSFPA desde seus mecanismos de identificação e criação (1991-1994), assim como dos resultados e efeitos de sua implantação e desenvolvimento (1995-2015), e inclusive sua revisão (2004-2017) e seu impacto, contribuindo com evidências empíricas qualitativas e quantitativas, de acordo com os indicadores de referência propostos pela Recomendação sobre o estabelecimento de um Quadro de Referência Europeu de Garantia da Qualidade na FP.
Keywords:

qualification, professional training, quality, evaluation, pharmaceutical industry

Palavras-chave: qualificação, formação profissional, qualidade profissional, qualidade, avaliação, indústria farmacêutica 


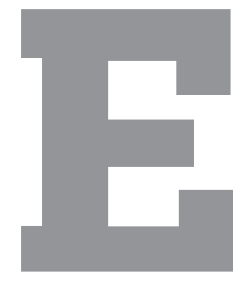

ntre los retos específicos para el sector de la educación superior en Europa figura: “... equipar a los estudiantes con mejores conocimientos, cualificaciones y competencias, y, al mismo tiempo, abordar la inadecuación de las cualificaciones y la escasez de las mismas en determinados sectores profesionales" (Consejo de la Unión Europea [CE], 2017, p. C 429/3)

Como un indicador del capital humano y del nivel de competencias de la población se suele utilizar el nivel educativo (Instituto Nacional de Evaluación Educativa [INEE], 2017), que es el porcentaje de una población que ha alcanzado un cierto nivel y posee una cualificación formal del mismo. En España no existía formación y por tanto titulaciones formales que respondieran a la Directiva Europea 91/356/CEE (CE, 1991), por la que se establecen los principios y directrices de las Normas de Correcta Fabricación de medicamentos (NCF), a fin de mantener altos estándares de Garantía de Calidad en el desarrollo, fabricación y control de medicamentos. La garantía incluye que los fabricantes están obligados a disponer de suficiente personal competente y debidamente cualificado para alcanzar el objetivo del sistema de calidad farmacéutica. También exige que el personal reciba formación inicial y permanente sobre la garantía de calidad y de NCF, así como su aplicación a las diferentes operaciones de producción.

Esa necesidad surge en el proceso de reflexión sobre el sistema educativo y la sociedad en España, que derivó en un nuevo modelo de formación profesional, dispuesto en la Ley Orgánica 1/1990, de Ordenación General del Sistema Educativo (LOGSE, 1990), en la perspectiva de la creación de un Sistema Nacional de Cualificaciones, primer objetivo del Primer Programa Nacional de Formación Profesional de 1993. El sistema incide en que la Formación Profesional (FP) tenga por objetivo inmediato la inserción laboral, lo cual implica que todos los alumnos que finalicen un ciclo formativo han adquirido una cualificación suficiente para trabajar con un determinado nivel de responsabilidad y autonomía. "La formación profesional específica facilitará la incorporación de los jóvenes a la vida activa, contribuirá a la formación permanente de los ciudadanos y atenderá a las demandas de cualificación del sistema productivo" (LOGSE, 1990, p. 28933)

\section{La formación basada en competencias}

La puesta en marcha en España de la formación basada en competencias, y su desarrollo en la formación profesional, permitió valorar la necesidad de que el sector farmacéutico en España contara con profesionales cualificados capaces de adaptarse a las innovaciones tecnológicas y organizativas. La industria farmacéutica, por su relación con la salud, tiene gran presencia de altos niveles de cualificación, en el que dominan las mujeres. Los laboratorios deben establecer y mantener un sistema de control de la calidad, y la fabricación es responsabilidad de una "persona cualificada"; esta es, en general, con licenciatura (Grado) en Farmacia o Química 
pero, siendo crucial, no es el objeto de la investigación sino los operadores de fabricación y acondicionado, así como sus responsables directos, supervisores, donde se había identificado el vacío formativo.

En el marco del proyecto de reforma de la formación profesional y en la familia profesional Química, la autora de este artículo dirigió el diseño de los títulos, junto con expertos tecnológicos y formativos, sobre la base de un estudio sectorial, y de acuerdo con una metodología, que condujo en 1993 a la creación del título de Técnico en Operaciones de Fabricación de Productos Farmacéuticos (TOFPF) (incluido en educación secundaria superior) y el Técnico Superior en Fabricación de Productos Farmacéuticos y Afines (TSFPFA) (educación superior, nivel no universitario).

El estudio precisó que el sector farmacéutico en Europa y en España es líder en I+D, productividad, exportaciones y empleo, además ofrece una fuente decisiva de puestos de trabajo de elevada cualificación y contribuye de manera significativa a alcanzar los objetivos económicos, sociales y de salud pública. Países como Francia, Inglaterra, Alemania o Italia, líderes también en producción de fármacos contaban, ya entonces, con cualificaciones bien estructuradas para el sector, tanto de nivel universitario como técnico. La carencia de cualificaciones oficiales en el segmento del trabajo técnico para la fabricación de productos farmacéuticos dificultaba el desarrollo de capital humano requerido para afrontar la regulación normativa, la innovación tecnológica y la internacionalización de las empresas.

\section{El sistema de cualificaciones}

Una vez implementado el aprendizaje basado en competencias, instaurado desde el final de los años 90 en la formación profesional de grado medio y superior del sistema educativo, quedaba pendiente, entre otros retos, la articulación de las distintas formas de aprender, fundamentalmente con la educación de los trabajadores ocupados y desocupados, regidos por la administración laboral. Además, era necesario poner en marcha procedimientos para reconocer los aprendizajes no formales e informales, e impulsar la calidad de la oferta formativa, así como mejorar su imagen y visión social. Para ello acordó el Gobierno implementó para los empleadores y los trabajadores un segundo Programa Nacional de Formación Profesional en 1998, con el objetivo de crear un Sistema de Cualificaciones, apoyado por el Instituto Nacional de las Cualificaciones (INCUAL).

El INCUAL, como instrumento técnico de carácter independiente, fue creado por el Real Decreto 375/1999 como órgano técnico de apoyo del Consejo General de Formación Profesional (CGFP) para establecer, entre otras funciones, las cualificaciones que constituyen el Catálogo Nacional de Cualificaciones Profesionales (CNCP). Con este apoyo se aprobó la Ley Orgánica 
5/2002, de 19 de junio, de las Cualificaciones y de la Formación Profesional (LOCFP), que creó el Sistema Nacional de Cualificaciones y Formación Profesional cuyo principal instrumento es el CNCP. Un sistema de cualificaciones (OCDE, 2008, p. 27):

Son todos aquellos dispositivos de un país que dan lugar al reconocimiento de una formación o un aprendizaje. Incluyen medios para diseñar y operar políticas nacionales o regionales de cualificaciones, disposiciones institucionales, procesos de garantía de la calidad, procesos de evaluación y titulación, reconocimiento de capacidades y otros mecanismos que vinculan el ámbito educativo-formativo con el mercado de trabajo y la sociedad civil. Los sistemas de cualificaciones pueden ser más o menos integrados y coherentes. Un elemento de un sistema de cualificaciones puede ser un marco de cualificaciones.

El inventario global de marcos regionales y nacionales de cualificaciones (Cedefop, 2017) sistematiza hasta 154 países en todo el mundo que están desarrollando marcos nacionales de cualificaciones (MNC), como parte de sus sistemas de cualificaciones. Se han identificado cuatro elementos comunes en todos los sistemas: legislación, participación de los interesados, instituciones y aseguramiento de la calidad.

El artículo 7 de la LOCFP (Ley Orgánica 5/2002) define Cualificación profesional como "el conjunto de competencias profesionales con significación para el empleo que pueden ser adquiridas mediante formación modular u otros tipos de formación y a través de la experiencia laboral" (p. 22439). Las cualificaciones se acreditan mediante títulos del sistema educativo y certificados de profesionalidad de la administración laboral. La transposición de la Directiva europea sobre reconocimiento de cualificaciones profesionales (Real Decreto 581/2017), define Cualificación profesional como

Capacidad para el acceso a una determinada profesión, o a su ejercicio, que viene acreditada oficialmente por un título de formación, por un certificado de competencia tal como se define en el artículo 19.1.a), por una experiencia profesional formalmente reconocida, o bien por el concurso de más de una de tales circunstancias (p. 48163).

El Marco Europeo de Cualificaciones para el aprendizaje permanente (CE, 2017), en su anexo 1 define la cualificación como "resultado formal de un proceso de evaluación y validación que se obtiene cuando una autoridad competente establece que una persona ha alcanzado los resultados de aprendizaje correspondientes a unas normas determinadas"(p. C189/20).

El Catálogo se ordena verticalmente en cinco niveles de cualificación y de forma horizontal en 26 familias profesionales. Los cinco niveles de cualificación en los que seestructura el Catálogo atienden a la competencia profesional requerida por las actividades productivas con arreglo a criterios de conocimientos, capacidades, iniciativa, autonomía, responsabilidad y complejidad, entre otros, de la actividad por desarrollar. Los niveles de cualificación se relacionan con el citado Real Decreto 581/2017, y con las clasificaciones de educación (CNED), de ocupaciones (CNO) y de grupos de cotización. Dentro de la familia profesional Química, dirigidos por el 
INCUAL, y con la participación de expertos de las organizaciones productivas y del entorno de la formación, se definieron a partir de 2005 nuevas cualificaciones profesionales de nivel 2 y 3 para la industria farmacéutica, que permitieron actualizar los títulos y crear certificados de profesionalidad.

El Sistema Nacional de cualificaciones implicó cambios normativos de gran calado para la acreditación de las cualificaciones profesionales del CNCP en forma de títulos del sistema educativo, como de certificados de profesionalidad de la administración laboral. Así fue aprobada la Ley Orgánica 2/2006, de 3 de mayo, de Educación (LOE), modificada en 2013 por la LOMCE, que ha permitido la renovación de los títulos establecidos en el marco de la LOGSE. En la formación profesional para el empleo se modificó también la normativa, constituyéndose el Repertorio de Certificados de Profesionalidad, vinculado directamente con el CNCP. Además, se estableció la normativa (2009), que permite el reconocimiento de los aprendizajes adquiridos por la experiencia laboral y vías no formales de formación, cuestión esencial para un sector donde no había existido cualificación y formación oficial previa a 1993.

Por otraparte, el títulodeTécnicoSuperior seinscribeenelMarcoEspañoldeCualificaciones de Educación Superior, MECES, establecido por Real Decreto 96/2014. Este marco se estructura en cuatro ciclos, el título de Técnico Superior, de carácter postsecundario no universitario, objeto de la investigación es, junto con otros tipos de títulos, el nivel 1 del MECES; el nivel 2 lo constituye el Grado; el nivel 3, el Máster; y el nivel 4 corresponde al Doctor. Cada nivel se caracteriza mediante descriptores genéricos basados en resultados del aprendizaje e incluye una cuantificación orientativa de los créditos.

\section{El objetivo de investigación}

La cuestión está en valorar si fue acertada la decisión de crear estas dos cualificaciones (Cedefop, 2015) a través de la utilidad del título, como reconocimiento formal, para encontrar trabajo o para proseguir los estudios, condicionada por los resultados del aprendizaje realizado por las personas que finalizaron el programa de formación y aprobaron las evaluaciones correspondientes. Para ello el artículo recorre la investigación de tesis doctoral sobre las "Cualificaciones Profesionales de la Industria Farmacéutica en el marco de la LOGSE" (Arbizu, 2015) en el período entre 1990 y 2015, a fin de valorar la calidad, pertinencia y eficacia de esas primeras cualificaciones, a través de sus mecanismos de identificación y desarrollo, así como de los resultados de su aplicación.

La relación entre la formación, la productividad y los salarios es una afirmación frecuente, pero demostrarlo con datos e indicadores exige un análisis e investigación de los mecanismos que conducen a esos efectos. En todos los países (OCDE, 2017), los cambios sustanciales en las necesidades de competencias están desafiando el mercado laboral y las políticas de formación. 
En la mayoría de los países, una gran parte de los empleadores se quejan de que no pueden encontrar trabajadores con las habilidades que su empresa requiere. Por otra parte, asegurar el acceso igualitario de todos los hombres y las mujeres a una formación técnica, profesional y superior de calidad, incluida la enseñanza universitaria, es un objetivo de la Agenda 2030 para el Desarrollo Sostenible (UN, 2015).

La hipótesis de investigación es coincidente con las finalidades que el precitado apartado 5 del artículo 30 de la LOGSE establecía de forma general para la formación profesional específica: Las cualificaciones profesionales acreditadas como título de Técnico en Operaciones de Fabricación de Productos Farmacéuticos y de Técnico Superior en Fabricación de Productos Farmacéuticos y Afines facilitan la incorporación de los jóvenes a la vida activa, contribuyen a la formación permanente de los ciudadanos y atienden a las demandas de cualificación del sistema productivo. El objetivo es evaluar la calidad de las cualificaciones profesionales acreditadas como títulos de TOFPF, y de TSFPFA, a través de sus mecanismos de identificación y desarrollo, así como de los resultados y efectos de su aplicación.

\section{MÉTODO}

\section{Diseño metodológico}

La investigación toma como referencia recomendaciones y criterios europeos de garantía de calidad de la educación y formación profesionales, y la posibilidad de obtención de datos fiables para la evaluación de la calidad de los títulos de Técnico y Técnico Superior en que este artículo se centra. Por ello, el diseño de la investigación se concreta en tres dimensiones:

a. Adecuación de la formación a las necesidades de cualificación y del empleo en la fabricación de productos farmacéuticos.

b. Calidad de la formación y la acreditación de competencias en fabricación de productos farmacéuticos.

c. Graduación, inserción laboral y transición educativo-formativa de los titulados en fabricación de productos farmacéuticos.

La evaluación (OCDE, 2010, p. 21) es la:

Apreciación sistemática y objetiva de un proyecto, programa o política en curso o concluido, de su diseño, su puesta en práctica y sus resultados. El objetivo es determinar la pertinencia y el logro de los objetivos, así como la eficiencia, la eficacia, el impacto y la sostenibilidad para el desarrollo.

La investigación aplica una metodología de evaluación de programas de las dos cualificaciones profesionales acreditadas como título de Técnico TOFPF y de Técnico Superior TSFPFA, con una selección de indicadores, para evaluar, sistemática y objetivamente los dos títulos de FP. 
Los resultados pueden proporcionar criterios, de acuerdo con esos indicadores y otras informaciones, acerca de la calidad de estos títulos, tanto en su concepción como en su implantación para la mejora de la cualificación del personal técnico del sector farmacéutico, y la promoción de la cultura de la evaluación, como instrumento de mejora continua y de transparencia que mejore el funcionamiento de la formación.

\section{Participantes}

Muestra de alumnado y egresados: es de tipo censal, está constituida por el universo de los matriculados, así como los que finalizan y titulan en España. En Grado Medio la muestra es de un total de 1788 matrículas, y de 559 Técnicos en Operaciones de Fabricación de Productos Farmacéuticos. En Grado Superior la muestra es de un total de 2379 matrículas, y de 975 Técnicos Superior en Fabricación de productos farmacéuticos y afines en España.

Muestra de encuestas de inserción laboral a titulados: Se considera el período 2005/2006 a 2008/2009, solo en la Comunidad Autónoma de Cataluña, y la muestra es de 378 encuestas realizadas a 105 titulados en TOFPF y 273 en TSFPFA.

Muestra de trabajadores de industria farmacéutica en proceso de acreditación de las competencias adquiridas mediante la experiencia laboral: Se consideran los datos de la Comunidad Autónoma de Cataluña, en el periodo 2007/08 a 2008/09, en una muestra de 73 y de 124 trabajadores que se sometieron al proceso de acreditación en los niveles de grado medio y superior, respectivamente.

\section{Instrumentos}

Dado el largo proceso de investigación 1990-2014, se ha utilizado una combinación de técnicas e instrumentos del ciclo de vida de las cualificaciones para la evaluación de la formación profesional de la industria farmacéutica. Estas técnicas e instrumentos se toman en función de los criterios e indicadores por analizar. Se combinan fuentes primarias con fuentes secundarias y el análisis de las mismas: Análisis normativo, estadística, entrevistas semiestructuradas, observación directa, cuestionario a centros de formación, cuestionario a empresas y organizaciones farmacéuticas, estudio de casos.

\section{PROCEDIMIENTO}

\section{El ciclo de garantía de la calidad en la formación profesional.}

El desarrollo de la reforma de la formación profesional de la LOGSE introdujo cambios muy profundos, tanto en la concepción del sistema como en su ordenación, lo que aconsejaba acometer una evaluación del sistema con una visión prospectiva, a fin de corregir las carencias existentes y, sobre todo, anticipar las adecuadas medidas correctivas. La realidad es que en 
España y hasta la fecha, no se ha abordado una evaluación de la formación profesional, y menos aún con carácter sectorial centrándose en unos determinados títulos. El problema que trata de resolver la investigación es comprobar si el trinomio de la relación entre empleo, cualificación y formación en la fabricación de medicamentos y productos afines mejora por la implantación de la formación asociada a las dos cualificaciones profesionales objeto de investigación.

Ahora bien, algunas Comunidades Autónomas establecieron un plan institucional de evaluación de la calidad de la formación profesional. Así, Cataluña ha desarrollado tres iniciativas de evaluación que aportan elementos de interés: Evaluación de la Formación Profesional Reglada, relativa al período 2001-2008, por el Consejo Superior de Evaluación del Sistema Educativo; El estudio anual sobre inserción laboral de las enseñanzas profesionales que, desde 2005, promueven el Departamento de Educación con el Consejo de Cámaras de Cataluña; El proyecto de Calidad y mejora continua que el Departamento de Educación y Universidades ha impulsado, desde el curso 1998-1999, mediante experiencias para la mejora de la gestión en los institutos de educación secundaria que imparten formación profesional específica.

Como marco dela evaluación delos programas TOFPF y TSFPFAseutilizala Recomendación del Parlamento Europeo y del Consejo de18 dejunio de 2009 sobreel establecimiento de un Marco de Referencia Europeo de Garantía de la Calidad en la Educación y Formación Profesionales (Recomendación sobre el EqaVET). Por otra parte se toman en cuenta los Principios de garantía de la calidad de las cualificaciones que forman parte de los marcos o sistemas nacionales de cualificaciones correlacionados con el Marco Europeo de Cualificaciones reflejados en el anexo IV de la Recomendación (CE, 2017). Además, se han tomado en cuenta los Criterios y directrices europeas relativas a la garantía de la calidad en la educación superior (ESG) (ANECA, 2015), acordados por los Ministros de Educación de los países involucrados en el Proceso de Bolonia. El Marco de referencia comprende un ciclo de garantía y de mejora de la calidad de cuatro fases:

La Planificación: conllevó la identificación de la necesidad de cualificaciones para la industria de fabricación de productos farmacéuticos y afines, mediante un estudio sectorial y el análisis de referentes internacionales de países del entorno que contaban con cualificaciones para la industria farmacéutica. Además, se realizó la formulación de los perfiles profesionales y la formación como resultados de aprendizaje con criterios de evaluación, según la metodología de elaboración de títulos en la familia profesional Química;

La Implementación: puesta en marcha de los ciclos formativos utilizando los recursos disponibles, centros de formación, profesorado y empresas para la formación en centros de trabajo, con el fin de alcanzar los resultados de aprendizaje expresados como capacidades y competencias;

La Evaluación: análisis de los resultados y efectos de la intervención, durante la implementación, con el objeto de aprender de la experiencia. La evaluación puede ser entendida 
como una fase del ciclo de la cualificación profesional y como una actividad que influye sobre todas las demás.

La Revisión: desarrollo de procedimientos de cambio para llegar a la actualización de las cualificaciones profesionales definidas para la industria farmacéutica.

Cada una de estas fases se basa en criterios de calidad y en descriptores indicativos que deben aplicarse al sistema y a los centros de formación profesional, así como a los niveles establecidos de cualificaciones. El ciclo de garantía de calidad proporciona un planteamiento sistémico de retroalimentación respecto de la calidad al combinar la evaluación interna y externa, la revisión y los demás procesos de mejora, con arreglo a análisis cualitativos y cuantitativos.

El informe sobre los progresos en materia de garantía de la calidad en la FP en la Unión Europea (CE, 2014) tras la adopción de la Recomendación del EqaVET concluye que ha contribuido al fomento en los países europeos de una cultura de la calidad en la EFP, así como a su aplicación práctica, pero todavía debe mejorar su aplicación y conexión con ESG y el Marco europeo de cualificaciones.

Las Conclusiones del Consejo sobre una agenda renovada de la UE para la educación superior (CE, 2017) reconoce los progresos a la hora de avanzar hacia el objetivo principal de Europa 2020 de al menos el 40 \% de las personas entre 30 y 34 años que finalicen la enseñanza superior o equivalente de aquí a 2020, pero es fundamental para garantizar que la educación superior sea de gran calidad y relevancia que se permita a los titulados prosperar tanto en el ámbito personal como profesional. La garantía de la calidad desempeña un papel importante a la hora de abordar retos tales como mejorar su adecuación al mercado de trabajo y a la empleabilidad de los jóvenes, aumentar su atractivo y el de facilitar el reconocimiento mutuo del aprendizaje adquirido en distintos países y a través de distintas vías educativas, de modo que se logre una mayor movilidad y una mejor respuesta a los desafíos económicos y sociales. La Nueva Agenda de Capacidades para Europa (CE, 2016) insta a trabajar juntos para reforzar el capital humano, la empleabilidad y la competitividad

\section{Evaluación longitudinal: 25 años de cualificaciones.}

Esta investigación es de carácter longitudinal que abarca desde la gestación de los títulos a su evaluación, pasando por su implementación, cuyo ciclo corresponde a 25 años, en el período comprendido entre 1990 a 2015. Conviene recordar que la autora del artículo fue responsable de la gestación y diseño de los títulos en el periodo 1990-1993, así como Directora del Instituto Nacional de las Cualificaciones desde su creación hasta fin de 2008 (Arbizu, 2012). Esta investigación se extiende al modo en que estos dos títulos se han traducido en las comunidades autónomas en diferentes ciclos formativos de grado medio (CFGM) y de grado superior (CFGS).

En realidad, la investigación evaluativa comienza con su inclusión en el curso 1999/2000 en diferentes centros. Analizar el proceso por el cual se implantaron los títulos de TOFPF y 
TSFPFA nos permitirá sacar conclusiones sobre la relación entre la administración educativa y los laboratorios farmacéuticos. Dicho vínculo resultará, a la postre, primordial para la formación de profesionales cualificados (recordar el módulo de formación en centro de trabajo) y para el reconocimiento de las cualificaciones de aquellos trabajadores que han adquirido sus competencias profesionales mediante la experiencia laboral.

El estudio cuantitativo de resultados de su aplicación en los jóvenes, los trabajadores y la industria de fabricación de productos farmacéuticos y afines se valora entre 1999/2000 hasta el curso 2014/15, cuando la oferta formativa sigue activa en Andalucía, Asturias y Cataluña. Son por tanto 25 años desde la conceptualización, y 16 cursos académicos los de aplicación de estos dos títulos, tiempo que parece lo bastante razonable para evaluar la pertinencia, calidad e impacto de los títulos.

\section{Evaluación de resultados y efectos. Criterios de evaluación.}

Los elementos que se persiguen de acuerdo con la terminología del análisis de sistemas, hacen referencia a:

- Los inputs. Recursos, alumnos, profesorado, equipos, etc.

- Los throughputs. El proceso de desarrollo de los ciclos formativos.

- Los outputs. El producto final que se persigue con la acción formativa, traducido en adquisición de conocimientos, habilidades y destrezas.

- Los outcomes. Los efectos producidos en los participantes y en el desempeño de su trabajo, así como en la organización en su conjunto, después de la realización de la acción formativa.

La metodología de evaluación se centra en los resultados (outputs) y en los efectos (outcomes) después de transcurrido un tiempo de los ciclos formativos, con el propósito de verificar la permanencia y consistencia de los cambios producidos en las personas, las empresas y la sociedad.

La investigación presta atención a la evaluación de impacto (Billorou, Pacheco \& Vargas, 2011), que indica si el proyecto tuvo un efecto en su entorno en términos de factores económicos, técnicos, socio-culturales, institucionales y medioambientales (OCDE, 2010). También se entiende como un proceso orientado a medir los resultados generados (cambios y causas) por las acciones formativas desarrolladas en el escenario socioprofesional originario de las mismas al cabo del tiempo (Ferrández Lafuente, 2006, p. 20). De ahí que en esta investigación se ha tratado de medir los resultados transcurrido cierto tiempo desde el comienzo de la implementación

De las definiciones de evaluación de impacto es necesario identificar tanto los cambios que se producen tras la implantación y desarrollo de estos programas de formación profesional en los beneficiarios, como la variedad de impactos que puede tener la intervención-económicos, 
sociales-, y que pueden ser tanto previstos (definidos entre los objetivos de la actuación por evaluar) como no previstos.

\section{RESULTADOS}

Los resultados obtenidos toman como referencia los objetivos en la investigación.

\section{Ajuste de los títulos TOFPF y TSFPFA a las necesidades de cualificación de la fabricación de productos farmacéuticos y afines}

La industria farmacéutica en Europa es el sector de alta tecnología con el mayor valor añadido por persona empleada, significativamente más alto que el valor promedio para las industrias manufactureras y de alta tecnología (European Federation of Pharmaceutical Industries and Associations [EFPIA], 2014). El sector farmacéutico representa una gran contribución a la balanza comercial en ámbitos con gran intensidad de alta tecnología e investigación y desarrollo y ofrece puestos de trabajo de elevada cualificación y contribuye de manera significativa a alcanzar los objetivos económicos, sociales y de salud pública. En España, la industria farmacéutica es líder en I+D, productividad, exportaciones y empleo. El estudio ha comprobado que en el periodo 1995-2013, en la fabricación de productos farmacéuticos (Ministerio de Industria, Energía y Turismo [Minetur], 2015), el Índice de producción se elevó de 6.104,035 a 12.833,479 miles de $€$, mientras que el empleo disminuyó levemente de 39.242 a 36.992. La Productividad (VA/ocupados) se ha elevado de 56,4 a 112,9, por tanto, el coste laboral unitario ha disminuido desde $67,9 \%$ a $51,3 \%$.

La competencia general del Técnico en Operaciones de Fabricación de Productos Farmacéuticos (TOFPF), es realizar todas las operaciones de proceso y control de las diversas fases de fabricación de productos farmacéuticos y afines, controlando el funcionamiento, puesta en marcha y parada de los equipos, en condiciones de seguridad, calidad y ambientales establecidas, responsabilizándose del mantenimiento de primer nivel de los equipos (Real decreto 816/1993, p. 24708). En el Técnico Superior la competencia es de organizar y participar en una línea o equipo de producción, controlando el proceso farmacéutico y su nivel de calidad, cumpliendo las normas de correcta fabricación, y supervisando el cumplimiento de las normas de seguridad y ambientales (Real decreto 810/1993, p. 23020). Asociado a este perfil, se encuentra una formación modular que se acompaña con una fase de formación en centros de trabajo, para dar respuesta a las necesidades de cualificación del capital humano, y en línea con las reformas del currículo en Europa (Cedefop, 2012).

Los títulos se relacionan con la clasificación de los trabajadores, del XVII Convenio colectivo general de la industria química (Dirección General de Empleo, Resolución de 26 de marzo de 2013). El Técnico Superior responde al grupo 5, donde se incluyen la realización de 
las funciones de integrar, coordinar y supervisar la ejecución de varias tareas homogéneas con la responsabilidad de ordenar el trabajo de un conjunto de colaboradores de una unidad funcional de producción o envasado, con vigilancia de instalaciones y seguimiento de procesos. El Técnico responde a los niveles 3 y 4. La importancia de analizar esta relación se encuentra en que es la base de las retribuciones salariales y las condiciones de trabajo. Es un sector sin conflicto laboral, con estabilidad en el empleo y dignas retribuciones.

En 2017 genera unos 200.000 empleos en España, de los que más de 37.000 son directos. Es un empleo de calidad pues el $96 \%$ de los trabajadores de la industria farmacéutica tienen contrato indefinido y de igualdad y casi el 50\% del total son mujeres.

\section{Implantación de los ciclos formativos en Comunidades Autónomas con relación al entorno industrial}

La atribución que la LOGSE da a las Comunidades Autónomas para completar su propio currículo, a partir de las enseñanzas mínimas, ha conducido, en el caso del Técnico, a cuatro ciclos formativos muy diferentes desarrollados en Asturias, Andalucía, Navarra y Cataluña. El proceso de implantación de los ciclos formativos definidos para la fabricación de medicamentos se realiza por impulso, y en general a través de convenios, con las empresas farmacéuticas del entorno, sobre todo en Cataluña.

Si bien en el ámbito del Estado existen buenas estadísticas educativas, pero no un sistema de aseguramiento de la calidad, algunas Comunidades han establecido un plan institucional de evaluación de la calidad de la formación profesional. Cataluña ha desarrollado iniciativas de evaluación que aportaron elementos sustanciales en la investigación Por una parte la evaluación de la Formación Profesional Reglada, relativa al período 2001-2008, por el Consejo Superior de Evaluación del Sistema Educativo, según el Plan de Evaluación del Departamento de Educación, y las bases de 2008 (Generalitat de Catalunya, 2008). La evaluación no desciende a nivel de los ciclos formativos concretos. Además, el proyecto de Calidad y mejora continua que el Departamento de Educación y Universidades han impulsado, desde el curso 1998-1999, la mejora de la gestión en los institutos de educación secundaria que imparten formación profesional específica.

Se aplicó el Indicador No 1 EqaVET, Pertinencia de los sistemas de garantía de calidad para los proveedores de EFP. En el período 1999/2000 a 2014/2015 los ciclos formativos se han desarrollado en 10 centros de cuatro comunidades autónomas. El ciclo de grado medio de TOFPF, un centro en Andalucía (Almería), otro en Asturias, un tercero en Navarra (Pamplona), ya desaparecido y cinco en Cataluña (Barcelona). El ciclo de grado superior TSFPFA se desarrolla en seis centros de Cataluña, todos ellos en localidades de Barcelona. Todos los centros educativos de Cataluña cuentan con certificación de sistemas de garantía de calidad, ya sea por ISO 9001 o por EFQM. 
En la garantía de calidad de las cualificaciones cobra especial importancia el Indicador $\mathrm{N}^{\mathrm{O}} 2$ EqaVET, Inversión en la formación de profesores y formadores: proporción de profesores y formadores que participan en programas de formación complementaria, e importe de los fondos invertidos. Al ser unas enseñanzas nuevas la preparación del profesorado ya existente, en general químicos, era esencial. Las encuestas y entrevistas realizadas muestran que hubo formación inicial, y también continua, así como estancias en empresas, pero no de forma sistemática ni evaluada.

\section{Participación de alumnos en los ciclos formativos TOFPF Y TSFPFA}

Se usa el Indicador $\mathrm{N}^{\circ} 3$ de EqaVET, Tasa de participación en programas de EFP y el Indicador $\mathrm{N}^{\circ}$ 8. Prevalencia de grupos vulnerables, considerando a los participantes por género. La participación del alumnado en los ciclos de grado medio TOFPF en el periodo 1999/00 a 2013/14 ha sido 1997 matrículas. En el caso del grado superior de TSFPFA se alcanzó la cifra de 2694 con un ritmo de crecimiento positivo lento y continuo.

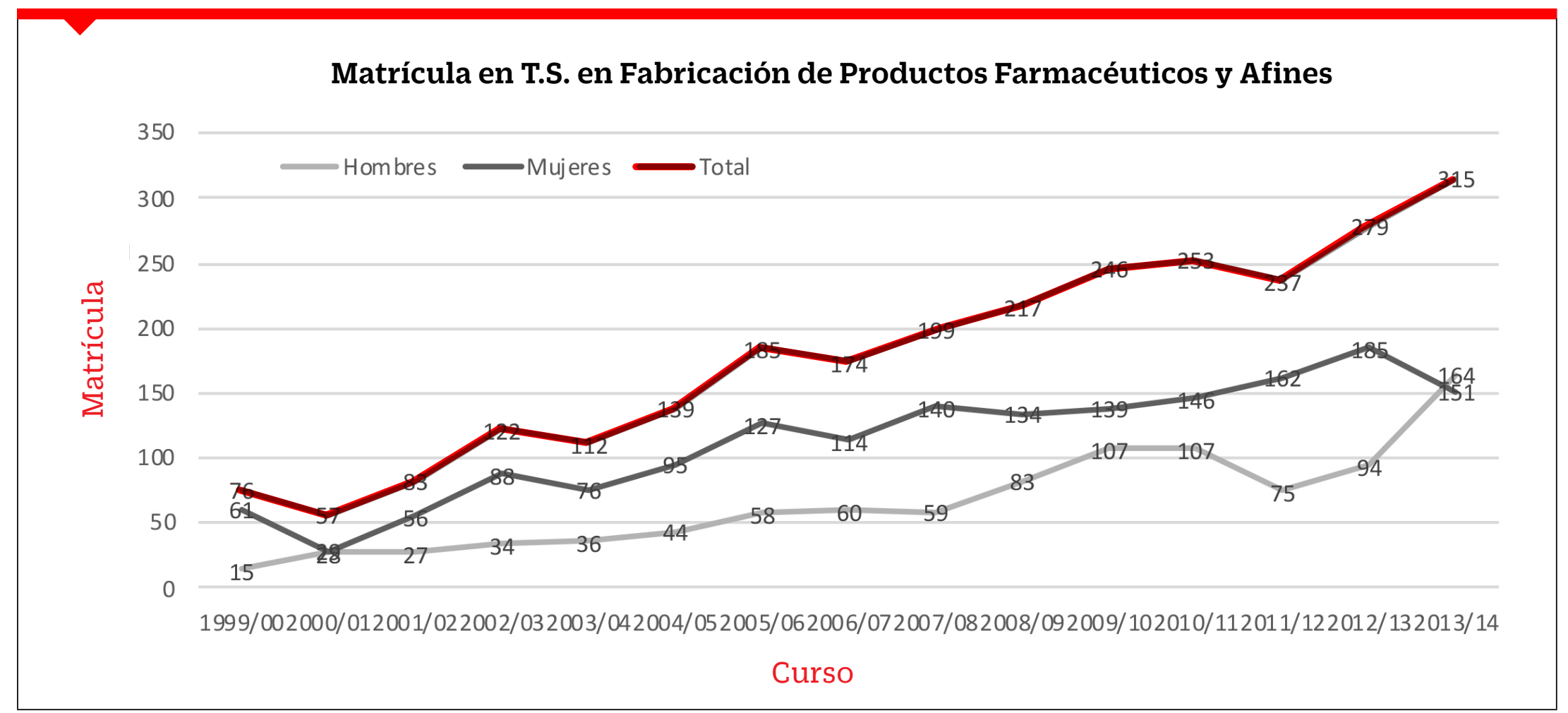

Figura 1. Matriculados en ciclo formativo de grado superior TSFPFA, 1999/00 a 2013/14

En ambos casos el número de mujeres es más del 63\% a la de hombres, sin embargo, esa diferencia era aún mayor en los primeros años de desarrollo de los ciclos por los convenios entre el Departamento de educación las empresas y los centros para la acreditación de las trabajadoras. Coincidiendo con la crisis económica se eleva la proporción de hombres. Por distribución geográfica domina Cataluña con más del 70\% de la matrícula en grado medio y $100 \%$ en grado superior. 


\section{Mejora del acceso a la formación en la gestión de los recursos humanos}

La estructura de los perfiles profesionales de cada título, organizados en unidades de competencia, ha permitido acreditar las competencias profesionales adquiridas por los trabajadores de la fabricación de productos farmacéuticos y afines mediante su experiencia laboral y formación continua. Se aplicó el Indicador $n^{\circ} 10$. Sistemas utilizados para fomentar un mejor acceso a la EFP. La existencia de pruebas de acceso a la formación para quienes no tienen los requisitos académicos de acceso fomenta la incorporación a la formación de grupos desfavorecidos fundamentalmente mujeres y trabajadores de cierta edad que se incorporaron al sector cuando no existía formación al respecto.

La combinación de los procedimientos de evaluación y acreditación de competencias con pruebas de acceso a la formación, para quienes no tienen requisitos académicos de acceso y una oferta de formación modular, ha permitido una elevación del nivel de cualificación de los recursos humanos de la industria farmacéutica.

El hecho de que existan títulos específicos para esta industria ha favorecido que empresas como Grifols se enfrenten a procesos de internacionalización en Estados Unidos donde se exige que el personal de fabricación esté acreditado. Los títulos han elevado el nivel de cualificación y son, en la práctica de empresas como Novartis, requisito en la selección de personal. Muchos titulados TOFPF preparan pruebas de acceso al ciclo de grado superior, y menos TSFPFA van a la universidad.

\section{Titulados Técnico y Técnico Superior de Fabricación de Productos Farmacéuticos y Afines}

Se relaciona con el Indicador $\mathrm{N}^{\circ}$ 4. Tasa de finalización de programas de FP: Número de personas que han finalizado con éxito/abandonado programas de FP. La titulación se consigue por la superación de todos y cada uno de los módulos profesionales que conforma el Ciclo Formativo, cuando el alumnado consigue una puntuación igual o superior a cinco puntos y apto en el módulo de Formación en Centros de Trabajo. La finalización o graduación también se promueve, en el caso de alumnos-trabajadores por la validación, acreditación y reconocimiento de competencias profesionales adquiridas por la experiencia laboral o vías no formales, y por exención del módulo de FCT.

En el periodo 2000/01 a 20013/14 se han titulado 630 Técnicos TOPFP con un 64\% de mujeres. En el caso de Técnicos Superiores hay 1046 TSFPFA, con 62\% de mujeres. El total de titulados, dotan de personal cualificado de acuerdo con las normas de correcta fabricación, y permite la renovación de las plantillas que tenía personal sin cualificación profesional acreditada, incompatible con el proceso continuo de innovación tecnológica e internacionalización. 


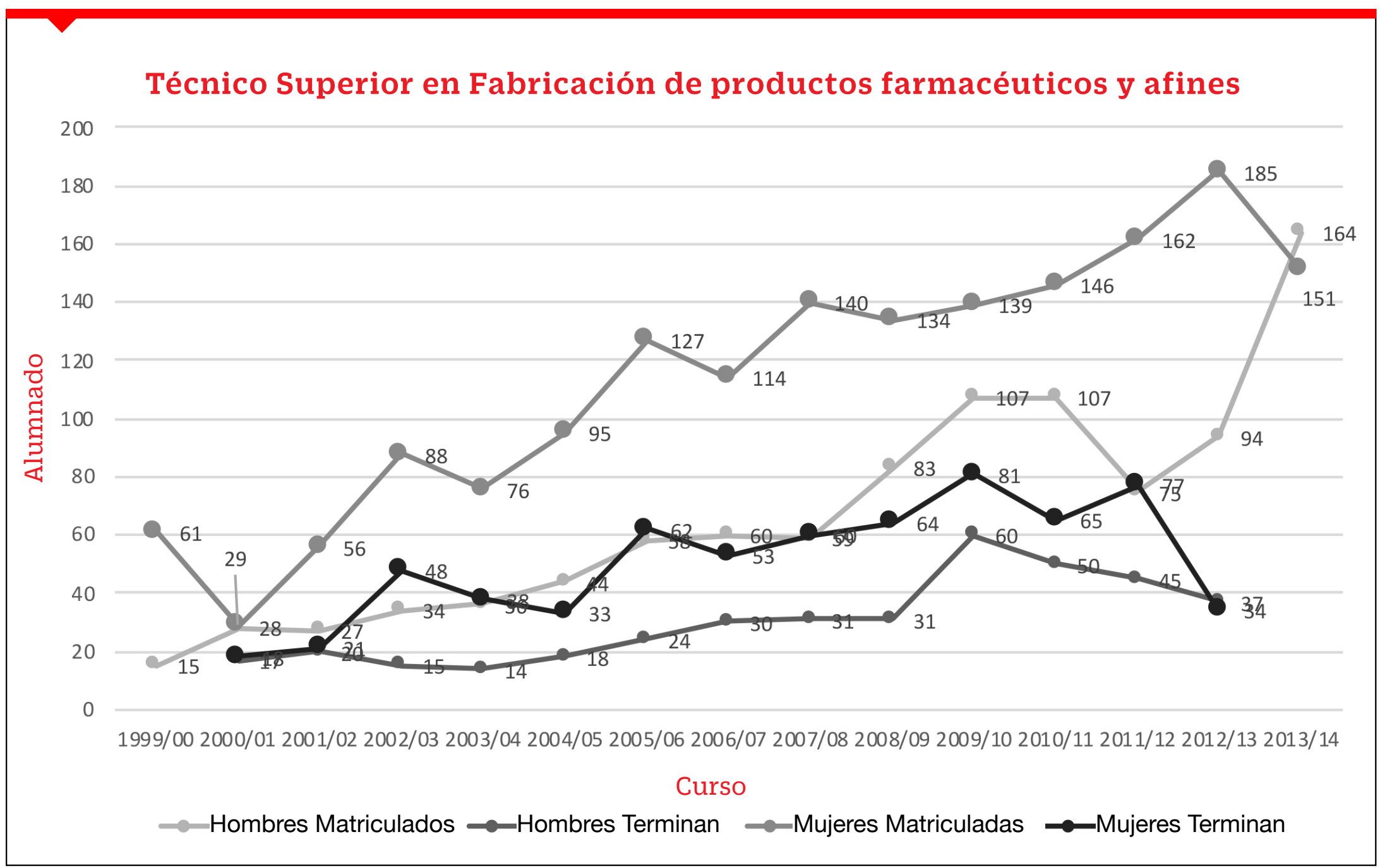

Figura 2. Alumnos matriculados y que terminan del TSFPFA, por género. Cursos 1999/00 a 2013/14

Los resultados de finalización de los ciclos formativos con respecto al alumnado . matriculado en los mismos, es del $63,09 \%$ en grado medio y $77,65 \%$ en superior, dondeincide la relación con la industria farmacéutica y cosmética, por participar gran número de trabajadoras que concilian su vida laboral con la formación gracias a la colaboración escuela-empresa. Ello hace que empleen más tiempo en la obtención del título, de modo que la ratio titulados/ matriculados baja.

\section{Inserción laboral y transición educativa de los titulados}

Se aplicó el Indicador $\mathrm{N}^{\circ}$ 5. Tasa de colocación en el marco de los programas de EFP y, complementariamente el Indicador $\mathrm{N}^{\circ}$ 7. Tasa de desempleo. Se utilizaron los resultados del estudio anual de Inserción Laboral de las Enseñanzas Profesionales, realizado por el Departamento de Enseñanza (Generalitat Catalunya, 2015) y el Consejo General de Cámaras de Cataluña, sobre los graduados como TOFPF y TSFPF desde 2006 hasta 2012/13 indican, en general y con cambios en el periodo por la crisis, una buena tasa de inserción laboral y con gran adecuación de los estudios al trabajo, siempre mayor en el técnico superior. El módulo de formación en centros de trabajo, inspirado en el sistema dual alemán de formación profesional fue el gran reto y una de las bases del éxito para la inserción laboral de los titulados. 
Los técnicos superiores que continúan estudiando, fundamentalmente en la universidad, son pocos frente a los Técnicos TOFPF que siguen estudiando, en este caso preparando la prueba de acceso a grado superior. Pocos están en paro en el momento de la encuesta, seis meses después de finalizar. Buena parte tiene contrato temporal y la mayoría están a jornada completa.

La remuneración se divide a medias entre los que ganan más de 1.500, los que cobran entre $1.200 €$ y $1.500 €$ y los que perciben entre 900 y 1.200 euros (10:40:40). Los datos de inserción están por encima de la media de Cataluña, y la calidad del empleo, en cuanto a la adecuación de los estudios y el trabajo realizado es también mejor, siendo reconocido el TSFPFA entre los cinco más demandados.

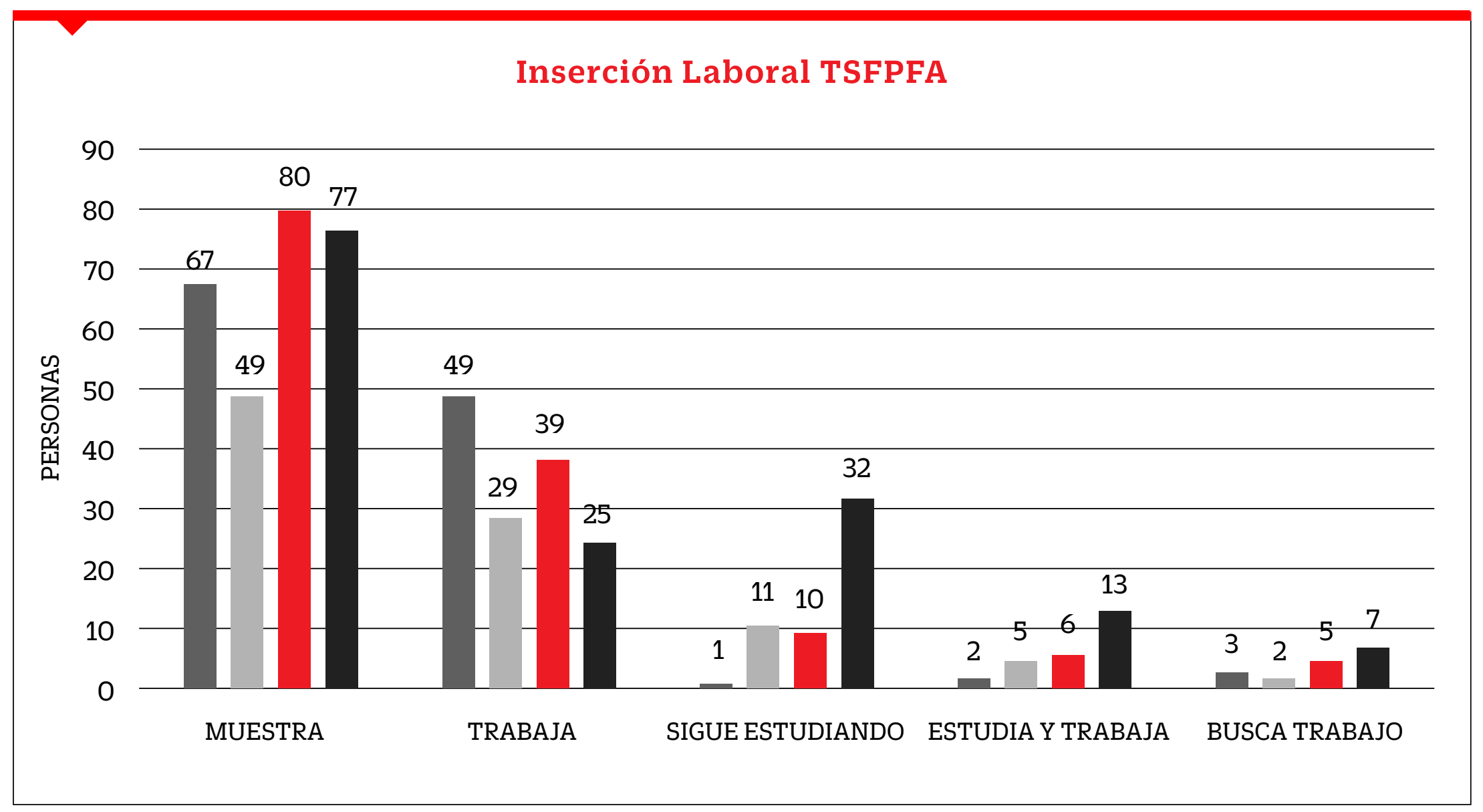

Figura 3. Inserción laboral y progresión educativa Titulados Técnico Superior, cursos 2005/2006, 2006/2007, 2007/2008 y 2008/2009

La inserción laboral a los seis meses de titularse en Cataluña es alta y de buena calidad. La formación en centros de trabajo lo apoya. Los titulados TSFPFA que van a la universidad convalidan hasta 33 créditos. Las salidas laborales están en relación con las ocupaciones y puestos de trabajo más característicos de TOFPF, el operador de especialidades y de acondicionamiento, y de TSFPFA, jefe de línea, jefe de equipo, encargado de fabricación o de acondicionamiento.

Se complementó con un estudio específico en el IES Narcis Monturiol que permitió aplicar el Indicador $\mathrm{N}^{\circ}$ 6. Utilización en el puesto de trabajo de las capacidades adquiridas. 


\section{Inserción Laboral y promoción educativa 2006/07}

Inserción Laboral General
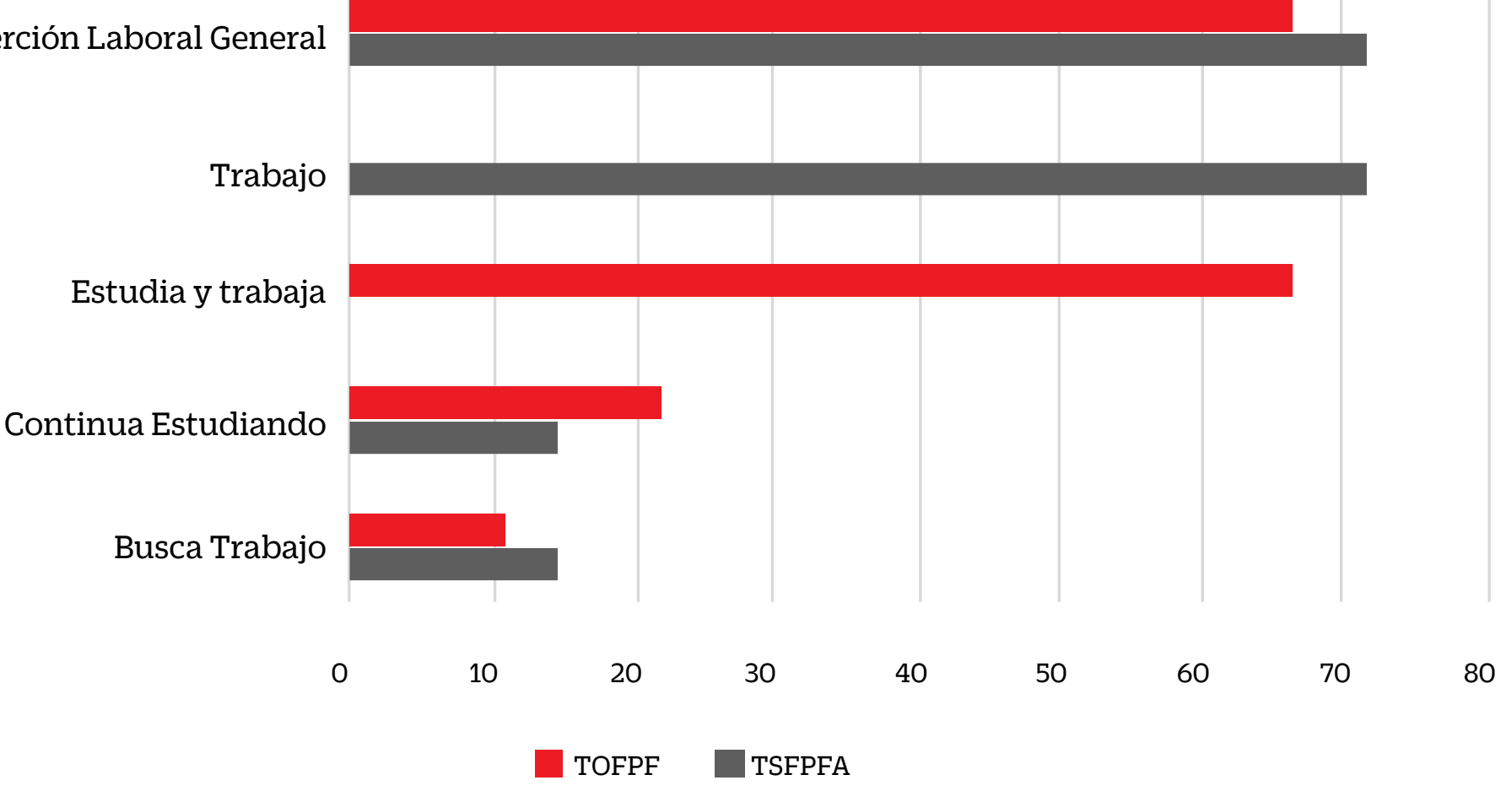

Figura 4. Inserción laboral y promoción educativa de alumnos del IES Narcis Monturiol

Este IES desarrolla siete ciclos formativos de Química, entre los que se encuentran los dos de Farmacia. Los resultados fueron muy positivos, sobre todo en el Técnico Superior por la alta empleabilidad y la calidad de la misma, en los estudios realizados por el centro en su gestión de calidad, sobre los alumnos en formación en centros de trabajo y egresados.

\section{La revisión de las Cualificaciones Profesionales}

La investigación cierra el círculo con la revisión de los dos títulos TOFPF y TSFPFA de 1993 en el marco de la LOGSE de 1990. Este resultado da respuesta al Indicador $\mathrm{N}^{\circ}$ 9. Mecanismos para determinar las necesidades de formación en el mercado laboral. La puesta en marcha del Sistema decualificaciones condujoala elaboración del Catálogo Nacional deCualificaciones Profesionales con expertos tecnológicos y formativos del sector y validado por las organizaciones sectoriales. Entre 2005 y 2011 se investigaron, diseñaron y establecieron cinco nuevas cualificaciones profesionales. Dos de ellas son de nivel 2: Elaboración de productos farmacéuticos y afines y Operaciones de acondicionado de productos farmacéuticos y afines, relativas al nivel de Técnico. Las otras tres cualificaciones profesionales son de nivel 3: organización y control de la fabricación de productos farmacéuticos y afines, organización y control del acondicionado de productos farmacéuticos y afines, y organización y control de procesos y realización de servicios biotecnológicos, relativas en el sistema educativo al Técnico Superior. 
Estas nuevas cualificaciones profesionales son la base de la nueva oferta formativa, integrada y articulada, que se acredita como títulos del sistema educativo y como certificados de profesionalidad. En el contexto de la actualizada Ley de Empleo y de la LOCFP 2002, en el periodo 2011-2013, han sido aprobados y publicados cinco Certificados de Profesionalidad de forma biunívoca con cada nueva cualificación profesional. Esta formación destinada a trabajadores ocupados y desempleados acredita de forma oficial y en todo el territorio del Estado las correspondientes cualificaciones profesionales.

Por su parte, la administración educativa con base en la Ley Orgánica 2/2006 (LOE, 2006) de Educación, modificada por la Ley Orgánica 8/2013 (LoMCE,2013) para la mejora de la calidad educativa, ha elaborado y establecido el nuevo título de Técnico Superior en Fabricación de productos farmacéuticos, biotecnológicos y afines, mediante el Real Decreto 832/2014, y en referencia a las tres cualificaciones profesionales de nivel 3.

El nuevo Técnico Superior ha ido sustituyendo al TSFPFA y extendiendo su radio de acción, pues ya está implantado en cuatro IES de Andalucía, Cataluña y País Vasco. El título de TSFPFA tiene los mismos efectos profesionales y académicos que el nuevo título de Técnico Superior. La oferta formativa es, por tanto, sostenible en el grado superior, lo que permite la formación en el futuro de jóvenes y trabajadores en la fabricación de productos farmacéuticos, afines y biotecnológicos, aunque no se asegura la trazabilidad entre las unidades de competencia y los módulos profesionales.

El ciclo formativo de grado medio de TOFPF en el curso 2017/2018 sigue impartiéndose en cinco Institutos de Educación Secundaria de Cataluña, según el Registro de centros educativos, si bien el Real Decreto 832/2014 preveía que desapareciera a partir de 2016. Hasta el momento no se ha aprobado un título de Técnico que pudiera sustituir al TOFPF, que es dominante en el sector.

Un resultado fundamental es que las cinco cualificaciones del CNCP posibilitan el reconocimiento de las competencias adquiridas por la experiencia laboral y vías no formales de formación, a partir de la aprobación del Real Decreto 1224/2009, lo que favorece la obtención de los títulos.

\section{DISCUSIÓN}

La investigación ha tenido, en su conjunto, los efectos esperados coincidentes con el mandato de la LOGSE, 1990, y con el Sistema Nacional de Cualificaciones, creado por la Ley Orgánica 5/2002, pues los resultados expuestos sobre las dos cualificaciones profesionales acreditadas como Técnico en Operaciones y Técnico Superior en Fabricación de Productos Farmacéuticos y Afines demuestran que esos ciclos formativos facilitan la incorporación de los jóvenes a la vida activa, contribuye a la formación permanente de los ciudadanos y atiende a las demandas de cualificación del sistema productivo. 
Los sistemas de garantía de la calidad sustentan la generación de confianza en las cualificaciones. Estas afirmaciones de que es necesario que las cualificaciones sean pertinentes, se sustenten en la calidad y den buenos resultados, son muy comunes en la literatura científica. Sin embargo, son pocas las investigaciones que puedan demostrarlo, pues es necesario un largo tiempo para que se puedan observar y medir los resultados de la educación y la formación en el mercado de trabajo y en la progresión y movilidad educativa.

Las principales conclusiones que se derivan de este estudio se sintetizan en los siguientes puntos:

1. La investigación cubre el ciclo de calidad de las dos cualificaciones profesionales pues comienza con su diseño y planificación (1990-1993) como formación basada en competencias en el marco de la LOGSE, siendo los primeros títulos de formación profesional para la industria farmacéutica en España, y evalúa sus resultados, de acuerdo a indicadores de referencia propuestos por la Recomendación Europea de Garantía de la Calidad EqaVET.

2. La industria farmacéutica es el sector de alta tecnología con mayor valor añadido por persona empleada, y en constante elevación en el período de estudio. Esto indica el acierto de preparar personal cualificado en el segmento técnico, como ya existía en países más desarrollados en el momento del comienzo de la investigación. Los títulos elaborados y estudiados se ajustan a las NCF.

3. El modelo de formación profesional enfocado a competencias adoptado en la LOGSE, la metodología utilizada con participación de expertos reconocidos del sector, y el uso de referencias internacionales son las claves que soportan el que el título de Técnico y el Técnico Superior, para la fabricación de productos farmacéuticos y afines, definidos en el año 1993, sigan vigentes y poco obsoletos en 2015, a pesar de las innovaciones tecnológicas y organizativas del sector. Los perfiles profesionales, estructurados en competencias, se ajustan a la demanda del sector farmacéutico y su formación contribuye a la mejora de la productividad de quien la adquiere. Este modelo, que es común a otros países da transparencia a las cualificaciones.

4. Los ciclos formativos TOFPF y TSFPFA son una oferta formativa pertinente y eficaz ante las demandas de conocimiento y competencias de las normas de correcta fabricación de la industria farmacéutica en el segmento del trabajo técnico de producción.

5. La estructura de los perfiles profesionales en unidades de competencia ha permitido acreditar las competencias profesionales adquiridas por los trabajadores de la fabricación de productos farmacéuticos, ya hayan sido adquiridas por la experiencia 
laboral, u otras vías de formación, y su acreditación facilita la obtención de alguno de los títulos TOFPF o TSFPFA.

6. Los títulos TOFPF y TSFPFA mejoran la gestión de los recursos humanos, pues acreditan cualificación profesional para la actividad de los operadores y supervisores defabricación de productos farmacéuticos que otorgan empleabilidad a sus titulados, llegando a constituirse como requisito de acceso al empleo y permiten el progreso en la educación y la formación permanente, la promoción y movilidad de los titulados.

7. La colaboración escuela-empresa se ha mostrado altamente beneficiosa para la elevación del nivel de cualificación, a través del módulo profesional de Formación en centros de trabajo, que se constituye en mecanismo de inserción laboral de calidad.

8. La puesta en marcha del Sistema de cualificaciones, que permite combinar la formación en instituciones de formación, en centros de trabajo, pruebas de acceso y acreditación de competencias adquiridas en las empresas farmacéuticas son una estrategia acertada de aprendizaje a lo largo de la vida. que ha favorecido la competitividad internacional con casos de éxito como el convenio entre la empresa Grifols y el IES La Románica.

9. La implantación de los ciclos formativos del TOFPF y TSFPFA es mayoritaria en Cataluña por la importancia del sector en ella. El desarrollo de mecanismos de evaluación de estas enseñanzas ha permitido concluir que los resultados de inserción laboral de los titulados en las empresas farmacéuticas son altos y de buena calidad. Además, hay una buena progresión educativa de los titulados, sobre todo los Técnicos hacia el Técnico Superior, a través de pruebas de acceso.

10. La oferta es sostenible, pues los títulos TOFPF y TSFPFA de 1993, en el marco de la LOCFP 2002, se actualizan a cinco Certificados de profesionalidad y al Técnico Superior en Fabricación de Productos Farmacéuticos, Biotecnológicos y Afines. El TOFPF se mantiene temporalmente, lo que pone en peligro la formación formal de los operadores de fabricación y acondicionamiento de medicamentos.

\section{RECOMENDACIONES}

De la investigación ha surgido un efecto no esperado, llegar a un modelo y metodología de evaluación sistemática y cíclica de los títulos TOFPF y TSFPFA en el sector farmacéutico, que puede servir para que los poderes públicos acometan esta labor esencial sobre los ciclos formativos de formación profesional, sobre todo por la implicación en el empleo. De este modo se podrá responder al compromiso de la Recomendación europea de garantía de calidad EqaVET, y realizar una evaluación de las cualificaciones y la formación, que permita afrontar los cambios profundos en las empresas, las organizaciones y el mercado de trabajo para constatar 
que la inversión en formación a lo largo de la vida es uno de los activos que permite afrontar con expectativas y garantías de éxito los retos de la globalización y la innovación tecnológica en las empresas, las organizaciones y el mercado de trabajo.

De forma holística, la investigación propone un sistema de indicadores, basado en recomendaciones europeas, para evaluar, sistemática y objetivamente, los títulos en consonancia con el ciclo de garantía de la calidad de formación profesional. Algunos de esos indicadores requieren de información estadística no disponible en el país, como en la formación del profesorado, lo que requiere mayor esfuerzo.

Se recomienda la aplicación de la Recomendación del Consejo de 2017, relativa al seguimiento profesional de los titulados de la educación terciaria (CE, 2017b), en el marco de la Comunicación sobre una Nueva Agenda de Capacidades para Europa, para mejorar la información estratégica y la documentación sobre la transición de estos titulados al mercado laboral y así tomar decisiones informadas con respecto a la formación y a las carreras profesionales.

Es necesario que el Ministerio de Educación en sus decisiones sobre los nuevos títulos de Técnico Superior se ajuste en mayor medida a las cualificaciones profesionales que toma como referencia, de acuerdo a la LOCFP, para así lograr mayor articulación con la oferta formativa del CNCP para los trabajadores y para la mejora de los efectos de la validación y reconocimiento de las unidades de competencia incluidas en las cualificaciones profesionales de referencia. Es recomendable, así mismo y deacuerdo con los resultados, que los poderes públicos responsables de las cualificaciones atiendan a los sectores estratégicos que no tienen muchos trabajadores, pero sí un gran valor añadido y mucha necesidad de personal cualificado, como sucede en la industria farmacéutica.

\section{REFERENCIAS}

Agencia Nacional de Evaluación, Calidad y Acreditación [ANECA] (2015). Criterios y directrices para el aseguramiento de Calidad en el Espacio Europeo de Educación Superior. Recuperado de http://www.enqa.eu/index.php/ home/esg/

Arbizu, F. M. (2012). 25 años de Cualificaciones, Formación Profesional y más. Formación XXI: Revista de Formación y Empleo, 13. Recuperado de https://goo.gl/XSXaL6

Arbizu, F. M. (2015). Cualificaciones profesionales de la industria farmacéutica en el marco de la LOGSE (Tesis doctoral). Recuperado de http://eprints.ucm.es/36115/1/T36918.pdf

Billorou, N., Pacheco, M. \& Vargas F. (2011). Guía para la evaluación de impacto de la formación. Montevideo: OITCinterfor.

Centro Europeo para el desarrollo de la Formación Profesional [CEDEFOP] (2012). Curriculum reform in Europe. The 
impact of learning outcomes. Luxembourg: Publications Office of the European Union, 2012

Centro Europeo para el desarrollo de la Formación Profesional [CEDEFOP] (2015). Ensuring the quality of certification in vocational education and training. Luxembourg: Publications Office of the European Union, 2015

Centro Europeo para el desarrollo de la Formación Profesional [CEDEFOP] (2017). Global inventory of regional and national qualifications frameworks 2017 (vol. I y II). Recuperado de https://goo.gl/8XtvZq

Comisión Europea [CE] (2014). Garantía de la Calidad en la Educación y Formación Profesionales (EFP). Recuperado de https://goo.gl/qyD958

Comisión Europea [CE] (2016). Una nueva agenda de capacidades para Europa. Trabajar juntos para reforzar el capital humano, la empleabilidad y la competitividad. Recuperado de https://goo.gl/rEA5xP

Consejo de la Unión Europea [CE] (2017). Conclusiones del Consejo sobre una agenda renovada de la UE para la educación superior. Recuperado de https://goo.gl/aoNWfx

Directiva 91/356/CEE de la Comisión, de 13 de junio de 1991, por la que se establecen los principios y directrices de las prácticas correctas de fabricación de los medicamentos de uso humano. Diario Oficial de las Comunidades Europeas, 17 de julio de 1991, L 193/30.

European Federation of Pharmaceutical Industries and Associations [EFPIA] (2014). The Pharmaceutical Industry in Figures. Key Data 2014. Bruselas: Autor.

Generalitat de Catalunya (2008). Document de bases de l'avaluació de la formació professional reglada a Catalunya 2008-2009. Barcelona: Autor.

Generalitat de Catalunya (2015). Inserció Laboral dels Ensenyaments Professionals 2009 - 2014. Recuperado de https:// goo.gl/CQxXnf

Ferrández Lafuente, E. (2006). La evaluación de impacto en el Master de Formación de Formadores CIFO-FLC: Informe de Investigación. Barcelona: Universidad Autónoma de Barcelona.

Instituto Nacional de Evaluación Educativa [INEE] (2017). Panorama de la Educación. Indicadores de la OCDE 2017. Informe Español. Madrid: Ministerio de Educación, Cultura y Deporte.

Ley Orgánica 1/1990, de 3 de octubre, de Ordenación General del Sistema Educativo. Boletín Oficial del Estado, 4 de octubre de 1990, 238.

Ley Orgánica 5/2002, de 19 de junio, de las Cualificaciones y de la Formación Profesional. Boletín Oficial del Estado, 20 de junio de 2002, 147.

Ley Orgánica 2/2006, de 3 de mayo, de Educación. Boletín Oficial del Estado, 4 de mayo de 2006, 106.

Ley Orgánica 8/2013, de 9 de diciembre, para la mejora de la calidad educativa. Boletín Oficial del Estado, 10 de diciembre de 2013, 295.

Ministerio de Industria, Energía y Turismo (25 de abril de 2018). Fichas sectoriales [Sitio web]. Recuperado de https:// goo.gl/s7kQ7N

Naciones Unidas [UN] (2015). Transformar nuestro mundo: la Agenda 2030 para el Desarrollo Sostenible. Recuperado de https://goo.gl/695i5q

Organización para la Cooperación y el Desarrollo Económico [OCDE] (2008). Sistemas de cualificaciones. Puentes para el aprendizaje a lo largo de la vida. Madrid: Instituto Nacional de las Cualificaciones. Ministerio de Educación, Política Social y Deporte.

Organización para la Cooperación y el Desarrollo Económico [OCDE] (2010). Glosario de los principales términos sobre evaluación y gestión basada en resultados. Recuperado de http://www.oecd.org/development/peerreviews/2754804.pdf

Organización para la Cooperación y el Desarrollo Económico [OCDE] (2017). Desarrollando las habilidades correctas: evaluar y anticiparse a los cambios en las necesidades. México: OCDE - ManpowerGroup.

Real Decreto 810/1993, de 28 de mayo, por el que se establece el título de Técnico Superior en Fabricación de Productos Farmacéuticos y Afines y sus correspondientes enseñanzas mínimas. Boletín Oficial del Estado, 28 de julio de 1993, 179 
Real Decreto 816/1993, de 28 de mayo, por el que se establece el título de Técnico en Operaciones de Fabricación de Productos Farmacéuticos y las correspondientes enseñanzas mínimas. Boletín Oficial del Estado, 13 de agosto de 1993, 193.

Real Decreto 375/1999, de 5 de marzo, por el que se crea el Instituto Nacional de las Cualificaciones. Boletín Oficial del Estado, 16 de marzo de 1999, 64.

Real Decreto 1224/2009, de 17 de julio, de reconocimiento de las competencias profesionales adquiridas por experiencia laboral. Boletín Oficial del Estado, 25 de agosto de 209, 205.

Real Decreto 96/2014, de 14 de febrero, por el que se modifican los Reales Decretos 1027/2011, de 15 de julio, por el que se establece el Marco Español de Cualificaciones para la Educación Superior (MECES), y 1393/2007, de 29 de octubre, por el que se establece la ordenación de las enseñanzas universitarias oficiales. Boletín Oficial del Estado, 5 de marzo de 2014, 55.

Real Decreto 832/2014, de 3 de octubre, por el que se establece el título de Técnico Superior en Fabricación de productos farmacéuticos, biotecnológicos y afines y se fijan sus enseñanzas mínimas. Boletín Oficial del Estado, 25 de octubre de 2014, 259.

Real Decreto 581/2017, de 9 de junio, por el que se incorpora al ordenamiento jurídico español la Directiva 2013/55/UE del Parlamento Europeo y del Consejo, de 20 de noviembre de 2013, por la que se modifica la Directiva 2005/36/ CE relativa al reconocimiento de cualificaciones profesionales y el Reglamento (UE) n. ${ }^{\circ}$ 1024/2012 relativo a la cooperación administrativa a través del Sistema de Información del Mercado Interior (Reglamento IMI). Boletín Oficial del Estado, 10 de junio de 2017, 138.

Recomendación del Parlamento Europeo y del Consejo de 18 de junio de 2009 sobre el establecimiento de un Marco de Referencia Europeo de Garantía de la Calidad en la Educación y Formación Profesionales. Diario Oficial de las Comunidades Europeas, 8 de julio de 2009, C 155/01.

Resolución de 26 de marzo de 2013, de la Dirección General de Empleo, por la que se registra y publica el XVII Convenio colectivo general de la industria química. Boletín Oficial del Estado, 9 de abril de 2013, 85.

RIDU / Revista Digital de Investigación en Docencia Universitaria / ISNN 2223-2516

(c) Los autores. Este artículo es publicado por la Revista Digital de Investigación en Docencia Universitaria del Área de Institutional Research and Effectiveness de la Dirección de Aseguramiento de la Calidad, Universidad Peruana de Ciencias Aplicadas. Este es un artículo de acceso abierto, distribuido bajo los términos de la LicenciaCreativeCommons Atribución-CompartirIgual 4.0 Internacional. ( http://creativecommons.org/licenses/by-sa/4.0/), que permite el uso no comercial, distribución y reproducción en cualquier medio, siempre que la obra original sea debidamente citada. 